\title{
Clinical, demographic, and radiographic analysis of 82 patients affected by florid osseous dysplasia: an international collaborative study
}

\author{
Débora Lima Pereira, DDS, MSc Student, ${ }^{\mathrm{a}}$ Fábio Ramôa Pires, DDS, PhD, ${ }^{\mathrm{b}}$ Márcio Ajudarte Lopes, DDS, PhD, ${ }^{\mathrm{a}}$ \\ Román Carlos, DDS, ${ }^{\mathrm{c}}$ John Marshal Wright, DDS, PhD, ${ }^{\mathrm{d}}$ Paras Patel, DDS, ${ }^{\mathrm{d}}$ \\ Willie van Heerden, BChD, MChD, FC Path(SA), PhD, DSc, ${ }^{\mathrm{e}}$ Andre Uys, BChD, MSc, ${ }^{\mathrm{e}}$ and \\ Pablo Agustin Vargas, DDS, PhD, FRCPath ${ }^{\mathrm{a}}$
}

Objective. The aim of this study was to correlate the clinical, demographic, and radiographic aspects of 82 cases of florid
osseous dysplasia (FOD) from four countries assessed by five reviewers.

Study Design. Retrospective data of patients diagnosed with FOD were obtained from archives of five institutes and analyzed according to clinical and radiographic criteria.

Results. A total of 82 cases of FOD were included in the study. In the majority (77.5\%) of FOD cases, black women were affected. The disease mainly affected the mandible (100\%), and the stage of the lesion was directly correlated with the age $(P<.05)$. The rates of convergence showed confluence of assessment in the mandible (posterior), and the highest rate of divergence between the reviewers was in assessing involvement in the maxilla. There were no differences among the characteristics of the lesion provided by the five institutions, except for the variable proportion of black patients affected by the disease.

Conclusions. FOD mostly affects the mandible of middle-aged black women, and its stage is correlated with patient age. Although panoramic radiography is used for diagnosis, the divergence in assessment of the maxilla might indicate that this method is not the most suitable to evaluate this area. (Oral Surg Oral Med Oral Pathol Oral Radiol 2016;122:250-257)

Osseous dysplasia (OD) represents a group of benign fibro-osseous lesions characterized by the replacement of normal bone by fibrous connective tissue with immature bone deposition that gradually becomes sclerotic, producing radiologic features varying from complete radiolucency to radiopacity. ${ }^{1}$ It affects mainly black women in the fifth to sixth decades of life, although it is also seen in whites and Asians. ${ }^{2-4}$

This group of dysplasia, which is subdivided into three variants-focal, periapical, and florid OD-affects mainly the jaws in the tooth-bearing or edentulous alveolar ridges. There is some evidence that OD arises from cells in the periodontal ligament tissue. ${ }^{1,5,6}$ These three variants share some similar clinical, histopathologic, and radiographic features,

The authors would like to thank the State of São Paulo Funding Agency (FAPESP grant: 2014/08930-0) and Carlos Chagas Filho Funding Agency of the State of Rio de Janeiro (FAPERJ) for their support.

${ }^{\mathrm{a}}$ Oral Diagnosis Department, Piracicaba Dental School, University of Campinas - UNICAMP, Piracicaba, Sao Paulo, Brazil.

${ }^{\mathrm{b}}$ Oral Pathology, State University of Rio de Janeiro, Rio de Janeiro, Rio de Janeiro, Brazil.

${ }^{c}$ Head and Neck Clinical Center, Ciudad de Guatemala, Guatemala. ${ }^{\mathrm{d} D e p a r t m e n t}$ of Diagnostic Sciences, Texas A\&M University Baylor College of Dentistry, Dallas, TX, USA.

e Department of Oral Pathology and Oral Biology, University of Pretoria, Pretoria, South Africa. except for location of the lesions and extension of maxillary involvement, with periapical OD occurring in the anterior mandible, focal OD in isolated areas except in the anterior mandible, and florid OD occurring multifocally, often in both the mandible and the maxilla. ${ }^{5,7,8}$

First described by Melrose et al. in $1976,{ }^{9}$ florid osseous dysplasia (FOD) is the most exuberant variant of OD. ${ }^{10,11}$ It commonly affects the posterior mandible, although it can also be seen in the maxilla and often presents bilaterally and in a symmetric pattern involving all four quadrants. ${ }^{7,12,13}$

Radiographically, FOD shows multifocal confluent radiolucency and mixed or radiopaque images surrounding the teeth root apices. The lesions have the tendency to increase in size and to become predominantly radiopaque. ${ }^{14}$ In the early stage, the radiographic appearance can mimic an inflammatory periapical lesion; therefore, it is important to consider FOD in

\section{Statement of Clinical Relevance}

Florid osseous dysplasia is a progressive disease that affects mostly black women; its evolution is correlated with the age of patients. Although panoramic radiography is the main examination used for diagnosis, it should be complemented with other more accurate modalities. 
Table I. Demographic and clinical data of 82 patients diagnosed with florid osseous dysplasia (FOD)

\begin{tabular}{|c|c|c|c|c|c|c|}
\hline & $\begin{array}{c}\text { Brazil } \\
(n=40)\end{array}$ & $\begin{array}{c}\text { Guatemala } \\
(n=3)\end{array}$ & $\begin{array}{l}\text { United States } \\
\quad(n=13)\end{array}$ & $\begin{array}{c}\text { South Africa } \\
\quad(n=26)\end{array}$ & Total & $P$ \\
\hline Mean age (years) & 55.6 & 45 & 53 & 52.92 & $53.9(27-79)$ & \\
\hline \multicolumn{7}{|l|}{ Gender $(\mathrm{n}=82)$} \\
\hline Male & 0 & 0 & 0 & $1(3.8 \%)$ & $1(1.2 \%)$ & \multirow[t]{2}{*}{.0001} \\
\hline Female & $40(100 \%)$ & $3(100 \%)$ & $13(100 \%)$ & $25(96.2 \%)$ & $81(98.8 \%)$ & \\
\hline \multicolumn{7}{|l|}{ Skin color $(\mathrm{n}=71)$} \\
\hline Black & $24(66.7 \%)$ & NA & $9(100 \%)$ & $22(84.6 \%)$ & $55(77.5 \%)$ & \multirow[t]{2}{*}{$.022^{*}$} \\
\hline Non-Black & $12(33.3 \%)$ & NA & $0(0 \%)$ & $4(15.4 \%)$ & $16(22.5 \%)$ & \\
\hline \multicolumn{7}{|l|}{ Infection $(\mathrm{n}=50)$} \\
\hline Yes & $15(40.5 \%)$ & NA & $3(23.1 \%)$ & NA & $18(36 \%)$ & \multirow[t]{2}{*}{$.047^{*}$} \\
\hline No & $22(59.5 \%)$ & NA & $10(71.9 \%)$ & NA & $32(64 \%)$ & \\
\hline \multicolumn{7}{|c|}{ Symptoms $(\mathrm{n}=51)$} \\
\hline Yes & $26(70.3 \%)$ & $1(100 \%)$ & $3(23.1 \%)$ & NA & $30(58.8 \%)$ & \multirow[t]{2}{*}{.207} \\
\hline No & $11(29.7 \%)$ & 0 & $10(71.9 \%)$ & NA & $21(41.2 \%)$ & \\
\hline \multicolumn{7}{|l|}{ Swelling $(\mathrm{n}=52)$} \\
\hline Yes & $18(46.2 \%)$ & NA & $4(30.8 \%)$ & NA & $22(42.3 \%)$ & \multirow[t]{2}{*}{.267} \\
\hline No & $21(53.8 \%)$ & NA & $9(69.2 \%)$ & NA & $30(57.7 \%)$ & \\
\hline
\end{tabular}

$N A$, not available.

$* P<.05$.

Table II. Chi-square test for symptom-age and symptom-treatment

\begin{tabular}{llcc}
\hline & \multicolumn{2}{c}{ Symptom } & \\
\cline { 2 - 3 } & \multicolumn{1}{c}{ No } & Yes & \\
\hline Age range (years) & & & \\
$27-40$ & $4(80 \%)$ & $1(20 \%)$ & .128 \\
$40-50$ & $8(50 \%)$ & $8(50 \%)$ & \\
$50-60$ & $5(35.7 \%)$ & $9(64.3 \%)$ & \\
$60-79$ & $4(25 \%)$ & $12(75 \%)$ & \\
Treatment & & & \\
$\quad$ No & $20(57.1 \%)$ & $15(42.9 \%)$ & .0006 \\
Yes & $1(7.1 \%)$ & $13(92.9 \%)$ & \\
\hline$* P<.05$. & &
\end{tabular}

the differential diagnosis of periapical radiolucencies and to be aware of tooth vitality, avoiding unnecessary endodontic treatment because teeth affected by FOD are vital. ${ }^{15}$

Because of the histologic similarity between fibroosseous lesions and the absence of swelling or symptoms, in general, FOD is diagnosed solely on the basis of clinical and radiographic correlation and coincidentally on routine radiographic examinations. ${ }^{6,16}$ Differential diagnosis should include familial gigantiform cementoma, the osteomas of Gardner syndrome, and Paget disease of bone, requiring other complementary examinations and the clinician's consideration. ${ }^{13,14,17}$ Additionally, some other lesions such as simple bone cysts have been described occurring concomitantly with FOD,${ }^{18,19}$ and, although rare, osteosarcoma can develop in association with a pre-existing FOD, making the differential diagnosis even more difficult and highlighting the importance of regular follow-up of the patients. $^{17}$
Although FOD is a well-known entity in most parts of the world, there is no international multicenter study correlating the main characteristics of the disease. The aim of this study was to correlate the clinical, demographic, and radiographic aspects of 82 cases of FOD from four different countries assessed by five reviewers.

\section{Materials and Methods}

This study was based on retrospective data of patients diagnosed with FOD obtained from archives of five institutes (Orocentro, Piracicaba Dental School, Brazil [1992-2014]; Laboratory of Oral Pathology, State University of Rio de Janeiro, Brazil [2005-2014]; Division of Pathology, Head and Neck Clinical Center, Guatemala [1997-2011]; Department of Oral Pathology and Oral Biology, University of Pretoria, South Africa [2012-2014]; The Texas A\&M University Baylor College of Dentistry, United States of America [2003-2014]). The Ethics Committee of the Piracicaba Dental School approved the present study (Protocol number: 043/14).

Five reviewers assessed 122 cases diagnosed as FOD by using panoramic radiographs. The radiographic features analyzed included affected sextant (right, central, and left maxilla; right, central, and left mandible); stage of the lesion (early, intermediate, or advanced); and number of teeth in the affected area. These criteria were correlated with clinical and demographic data (age, color of skin, gender, symptoms, treatment, infection, and swelling). The early stage was considered when the lesions in the periapical region were predominantly radiolucent; the intermediate stage was represented by mixed radiopaque or radiolucent 


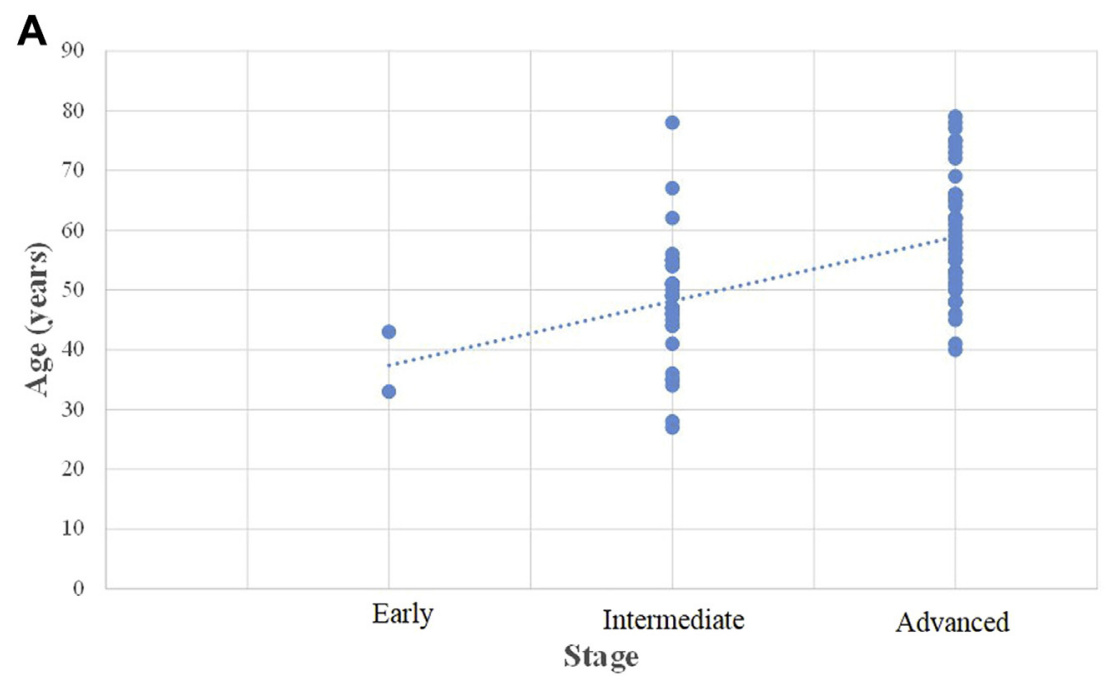

B

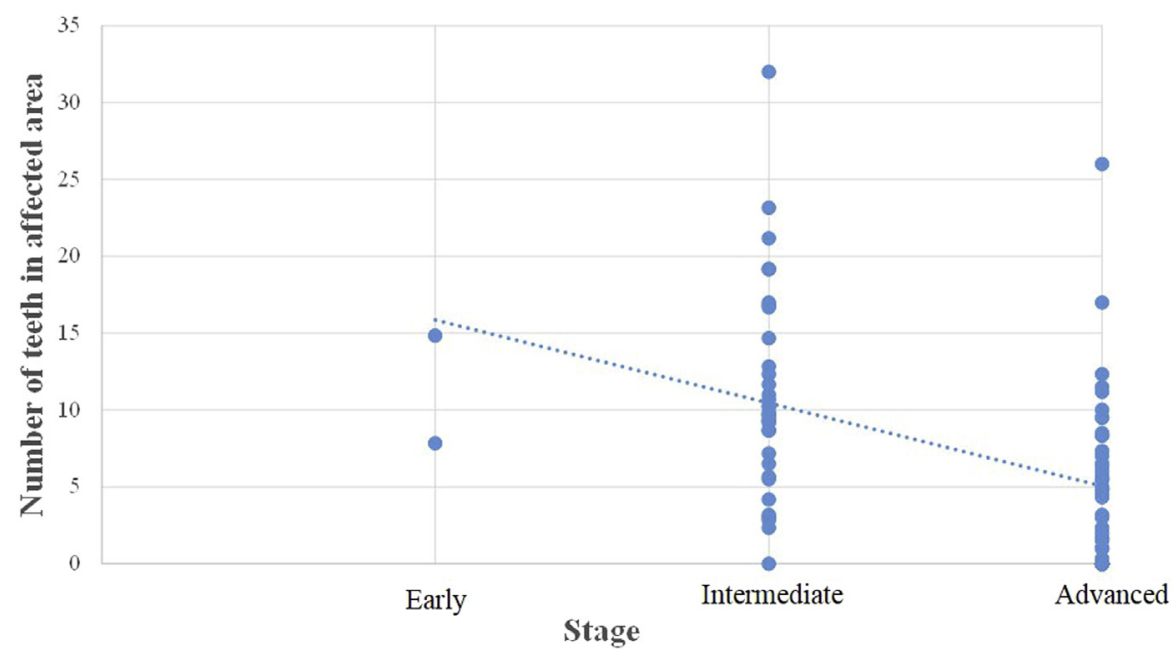

Fig. 1. Distribution of stage by age (A) and number of teeth in affected area (B). Stage $1=$ Early; Stage $2=$ Intermediate; Stage $3=$ Advanced.

images; and the advanced stage should present a prominent radiopaque image. All radiographs and information reflected the time of diagnosis. Cases in which at least one reviewer disagreed with the diagnosis were excluded from the study.

Statistical analyses were performed using the SAS system (SAS Institute Inc., Cary, NC, 2010). Bivariate correlations were assessed by the chi-square test and the correlation coefficient of Spearman. The analysis of variance between the five reviewers was established by Tukey's test. Interexaminer agreements were assessed using Cohen's kappa test to analyze the reliability of the examiners, and the agreement was considered reasonable or fair when kappa was between 0.20 and 0.40 , moderate or intermediate if between 0.40 and 0.60 , and substantial or strong when between 0.60 and $0.80 .^{20}$

\section{Results}

Among the 122 available FOD cases, 60 were from Brazil (Rio de Janeiro and Piracicaba), four from Guatemala (Guatemala City), 23 from the United States (Dallas), and 30 from South Africa (Pretoria). After the five reviewers analyzed all panoramic radiographs, 40 cases were excluded from the sample because of poor quality of images or disagreement with the diagnosis. The final sample consisted of 40 cases from Brazil, three from Guatemala, 13 from the United States, and 26 from South Africa. The mean age was 53.9 years (2779 years), and $98.8 \%$ were women. Blacks represented $77.5 \%$ of the sample, although this proportion differed significantly when separated by country $(P<.05)$. All demographic and clinical data are summarized in Table I.

Clinical data showed the presence of infection in 36\% out of 50 cases for which this information was available. 

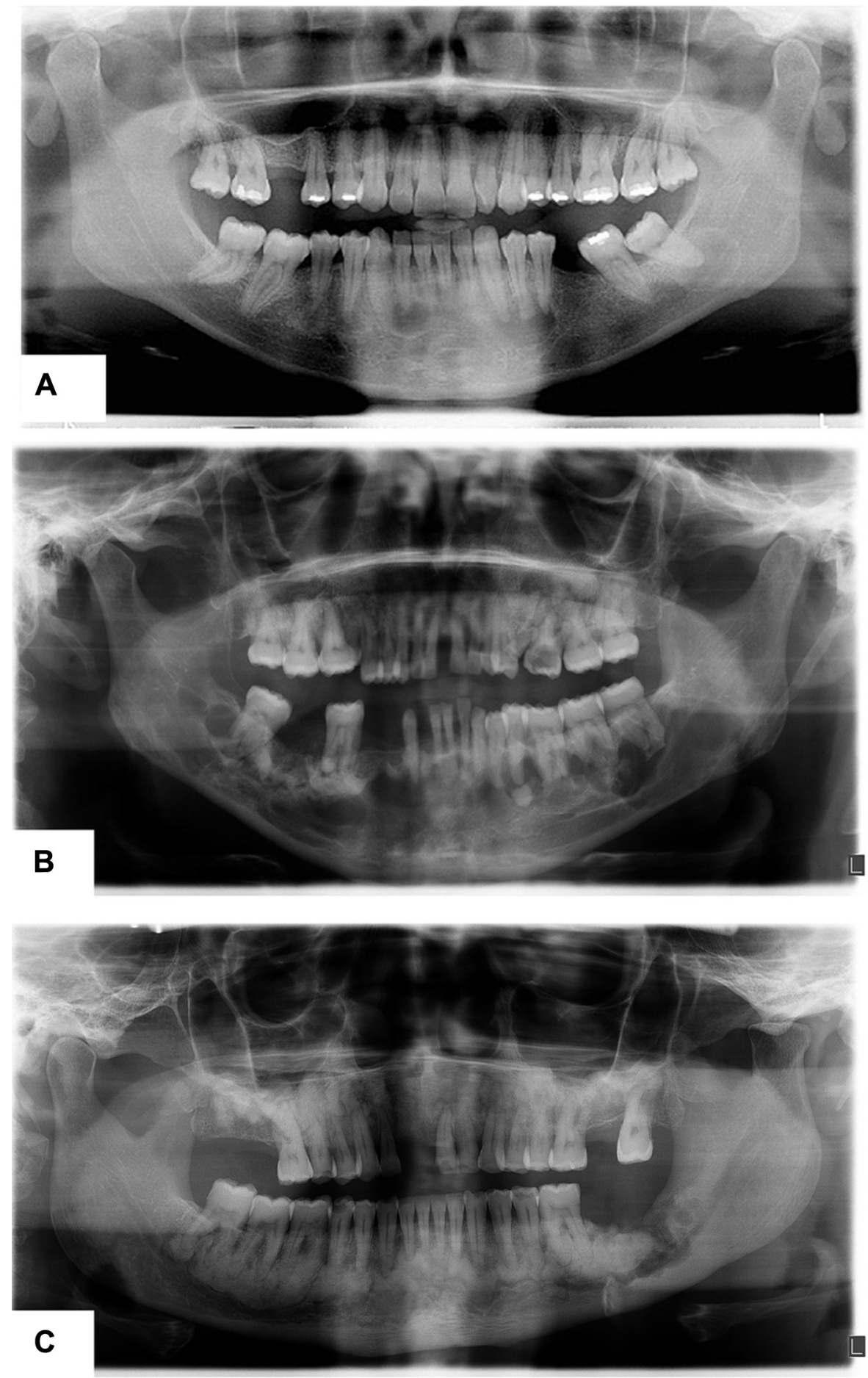

Fig. 2. Representative radiographies of three stages of florid osseous dysplasia (FOD). Early (A), intermediate (B), and advanced (C) stages.

However, this proportion varied significantly between Brazil and the United States $(P<.05)$. With regard to the chief complaint at the first appointment, 58.8\% $(\mathrm{n}=51)$ and $42.3 \%(n=52)$ of patients reported symptoms and swelling, respectively. Nevertheless, only 16 patients $(31.4 \% ; \mathrm{n}=51)$ received some type of treatment, such as antibiotics, surgery, or sequestrectomy $(P<.01)$ (see Table I). Additionally, $92.9 \%$ of treated patients were symptomatic $(P<.01)$ (Table II).

In spite of the absence of any significant correlation, the results also showed that as the age of patients increased, the percentage of patients complaining of 
Table III. Interexaminer agreements for stage of dis-ease evolution (early, intermediate, and advanced)

\begin{tabular}{cccc}
\hline & & \multicolumn{2}{c}{$95 \%$ Confidence interval } \\
\cline { 3 - 4 } Examiners & Kappa & Lower & Upper \\
\hline $1-2$ & 0.7825 & 0.6598 & 0.9052 \\
$1-3$ & 0.7212 & 0.5818 & 0.8607 \\
$1-4$ & 0.4180 & 0.2596 & 0.5763 \\
$1-5$ & 0.6935 & 0.5523 & 0.8348 \\
$2-3$ & 0.5891 & 0.4375 & 0.7408 \\
$2-4$ & $0.6438^{*}$ & 0.2042 & 0.4835 \\
$2-5$ & 0.67336 & 0.5163 & 0.8288 \\
$3-4$ & 0.5874 & 0.439 & 0.5933 \\
$3-5$ & 0.439 & 0.7350 \\
$4-5$ & $0.2918^{*}$ & 0.1613 & 0.4223 \\
\hline
\end{tabular}

*Fair agreement rate.

symptoms increased (see Table II). The increase in age was also correlated with more advanced stages, being considered positive and significant $(P<.05)$; however, it was negative when comparing age and stage with the number of teeth in the affected area (Figure 1A and 1B, respectively).

The stage of the disease showed two cases in the early stage $(2.4 \%), 34$ in the intermediate stage $(41.5 \%)$, and 46 in the advanced stage (56.1\%) (Figure 2A, 2B, and 2C, respectively). The interexaminer agreements applied to assessment of the stage of the disease were considered moderate or substantial in most combinations, varying from $41.8 \%$ to $78.3 \%$. The analysis showed fair agreement in only two cases, represented by kappa values of $29.2 \%$ and $34.4 \%$ (Table III).

The analysis of divergence between the examiners opinions on the presence of FOD involvement showed lower rates in the posterior region of the mandible compared with the maxilla and the anterior mandible $(P<.05)$ (Figure 3A). The convergence analysis demonstrated greater agreement between the examiners in the posterior mandible (Figure 3B). The mandible was affected in all cases, and the maxilla was involved in 43 out of 82 cases (52.4\%) (Figure 4).

\section{Discussion}

FOD is a well-known entity characterized by replacement of healthy bone by sclerotic bone. 7,8 The diagnosis is mainly based on clinical and radiographic aspects because their histologic features are similar to other fibro-osseous lesions. 6,21 In general, the condition presents no obvious signs and symptoms, and the diagnosis is established in middle-aged black women as an incidental finding on panoramic radiographs. 13 The present study evaluated 82 cases diagnosed as FOD and showed a prevalence of $98.8 \%$ for women, which is in accordance with published evidence. ${ }^{4}$ The mean age at diagnosis found in this study was 56 years but varied from 27 to 79 years, similar to previous findings. ${ }^{4}$ The main cause remains unknown, but some genetic or hormonal influences have been suggested 8 ; therefore, further studies are necessary to clarify this correlation.

In an extensive systematic review including 158 cases of FOD from 17 series, MacDonald-Jankowski ${ }^{4}$ showed that $59 \%$ of patients were black, $37 \%$ Asian, and $3 \%$ white. In contrast, the present study reported a rate of $77 \%$ of black people, but it varied according to the country. Brazil was the country with more miscegenation, presenting a rate of $66.7 \%$ of blacks, in contrast to the United States and South Africa, where blacks were affected at rates of $100 \%$ and $84.6 \%$, respectively. Curiously, except for South Africa, the southeastern and southern regions of Brazil and the entire United States have a significant population of Asian descendants, but this study did not show any case in this specific subgroup. A recent study conducted in Uganda showed 16 new cases of FOD and called attention to the lack of information about osseous dysplasia in sub-Saharan Africa. ${ }^{22}$ Although the disease has an evident predilection for black people, cases of FOD might be underreported or underdiagnosed in that region because of the frequent asymptomatic presentation.

In general, FOD is an indolent disease discovered accidentally on routine radiography. Occasionally, patients can present with symptoms, such as pain or swelling and purulent discharge, because of infection in the affected bone; treatment is usually antibiotic therapy with or without surgical debridement and removal of the affected bone. Given the poor vascularization of the advanced FOD lesions, the risk of infection, sequestrum formation, and osteomyelitis increases; therefore, elective bone manipulations, such as biopsies and teeth extractions, should be avoided or (when necessary) carefully planned. ${ }^{11,23}$ In this research study, 18 out of 50 patients presented with infection and 30 out of 51 patients had symptoms; among the 52 patients, 22 presented with swelling at the time of clinical exami-nation. It is important to mention that only 16 patients received some type of treatment, and among those, only one case was asymptomatic, which reinforces the notion that interventions in FOD should be reserved only for necessary cases.

Comparing the two studies of MacDonaldJankowski, the study including 17 cases of FOD diagnosed by histologic patterns showed the mean age was higher (62.1 years) than in the study of 16 cases diagnosed only by clinical and radiographic features (52.1 years). 24,25 This difference might be explained by the evolution of the disease because, as shown in the data from the present study, the stage of the disease is directly correlated with age, and with increasing age, 


\section{A}

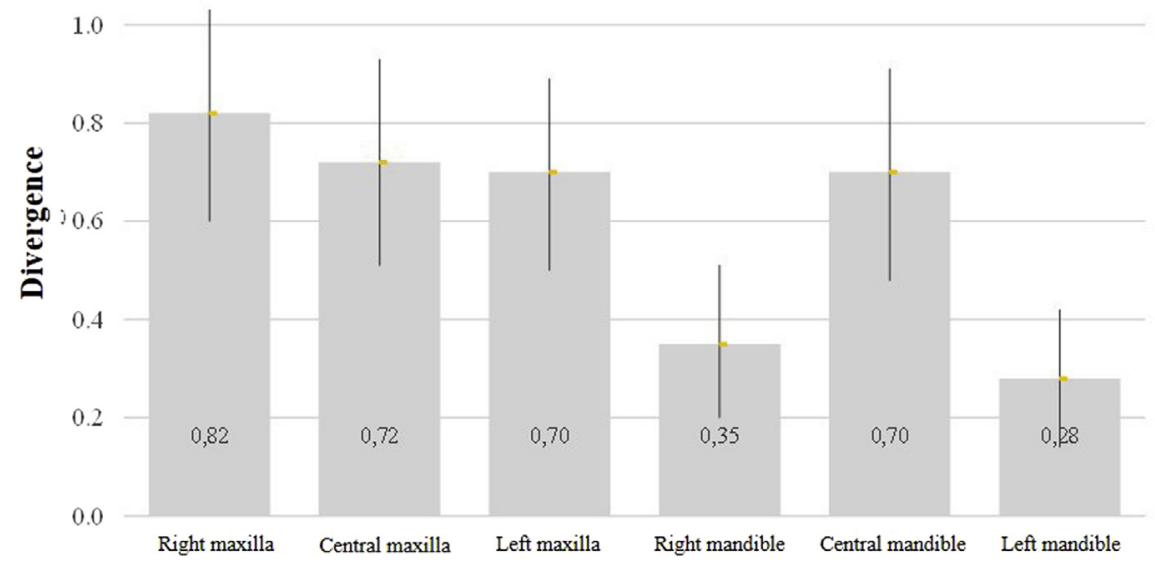

Sextant

\section{B}

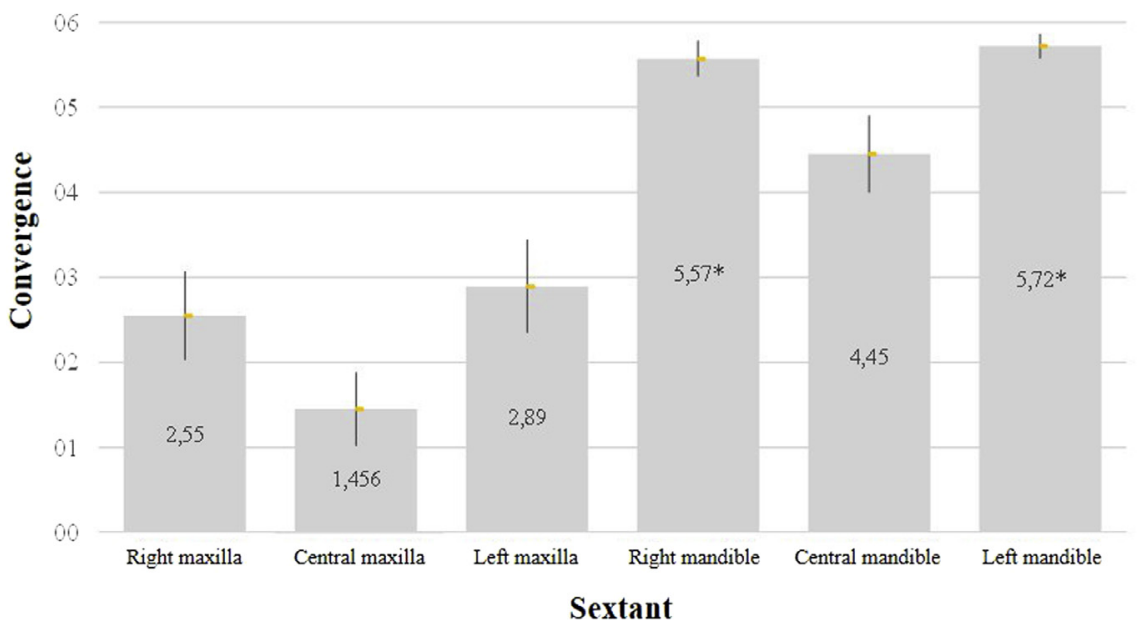

Fig. 3. Affected sextants. A, Divergence of agreement. B, Convergence agreement. ${ }^{*} P<.05$.

more patients become symptomatic, necessitating antibiotics and surgical intervention. Also important, the relation between age and the number of teeth was shown to be inversely proportional, which means that the absence of teeth in areas affected by OD might contribute to bone resorption. As bone affected by OD is compacted and poorly vascularized, absence of teeth, masticatory forces, and prosthesis trauma might facilitate bone exposure and, consequently, the development of infection and symptoms. However, affected areas with teeth should also be considered prone to infections because of the possibility of periodontal disease, periapical lesions, or tooth extraction.

Radiographically, FOD can present initially as a totally radiolucent lesion, which characterizes the early stage of the lesion, before changing to a mixed radiolucent and radiopaque appearance, called the intermediate stage, and finally becoming entirely radiopaque, usually with a radiolucent rim, reaching the advanced phase of the disease. ${ }^{5,21}$ Most cases $(97.7 \%)$ included in the present study were diagnosed in either the intermediate stage or the advanced stage. This feature could be associated with the fact that diagnosis is achieved when patients seek conventional dental treatment and need panoramic radiography for treatment planning or may be related to the difficulty in identifying radiolucent lesions as a characteristic FOD. Additionally, FOD may be diagnosed at these stages because symptoms tend to occur in the intermediate and advanced stages, leading the patient to look for treatment. 


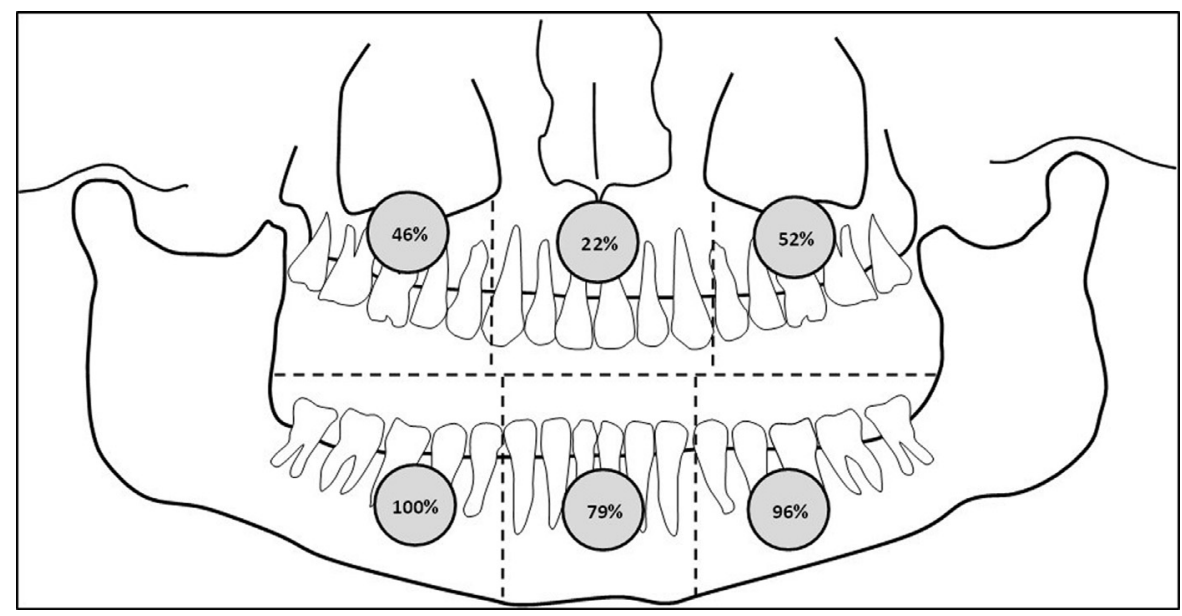

Fig. 4. Distribution of the frequency of florid osseous dysplasia (FOD) lesions by sextant.

In 2011, Alsufyani et al. emphasized the importance of correctly diagnosing osseous lesions. ${ }^{6}$ Their study, which was based on standardized imaging examinations, showed that agreement regarding the diagnosis of OD among maxillofacial radiologists was substantial (80\%-84\%), but agreement between general dentists was only slight to moderate (13\%$58 \%){ }^{6}$ In the present study, interexaminer agreement, as assessed by the kappa coefficient, was considered moderate or substantial for this criterion in most of the combinations (41.8\%-78.3\%); therefore, the analysis was satisfactory, mainly considering that the radiographs came from different institutes and were of varying quality, which might interfere with the analysis.

Although FOD can affect any tooth-bearing area, it is more common in the mandible, symmetrically. ${ }^{13}$ MacDonald-Jankowski, in his systematic review in 2003 , showed that $100 \%$ of cases affect the mandible, but a lower rate $(61.4 \%)$ was seen in the maxilla. This is consistent with the results of our study, in which all patients were affected in the mandible and about half of the patients presented with lesion(s) in the maxilla $(52.4 \%) .^{4}$ Moreover, the prevalence of the sextant affected seems to be higher in the posterior regions, especially in the mandible. Divergence analysis demonstrated high rates in all of the maxilla and the anterior mandible, which might be explained by the difficulties in assessing these regions resulting from the superimposition of the overlying anatomic structures.

Panoramic radiography is considered a satisfactory imaging modality for diagnosing most cases of FOD. In some challenging and indistinguishable cases, it might be necessary to use additional imaging modalities, such as periapical radiography, computed tomography, and cone beam computed tomography. ${ }^{13}$ Forty cases were excluded from the present sample because of the differences in the diagnosis given by the examiners. The reasons included difficulty in distinguishing some areas of involvement, suggesting the differential diagnosis of FOD and disagreement if some radiopaque lesions truly represented areas of OD.

Although biopsy should be avoided in cases of FOD because of the increased of the risk of infection, ${ }^{11}$ some cases require pathologic confirmation because of the aggressive behavior of the lesion. Of the 82 cases, one presented with a large painful growth in the mandible, and the lesion was biopsied and diagnosed as osteosarcoma. ${ }^{17}$ In this specific case, the biopsy was crucial to confirm the possible malignant transformation of this benign disease. Although only a few cases of development of malignant tumor in association with FOD are described in the literature, it is important to be aware of this possibility and maintain rigorous follow-up of all these patients..$^{17,26,27}$

\section{Conclusion}

In summary, FOD is a gradually progressive disease that affects mostly black women worldwide, and its evolution is directly correlated with the age of the patient. Accordingly, the disease followed the same pattern among different countries, except for the variable distribution of ethnicity. Because of high levels of divergence in the assessment of some difficult areas, such as the maxilla, panoramic radiography should be complemented by other more accurate examinations. It is recommended that surgical procedures be reserved exclusively for symptomatic cases. Dentists should stress the importance of adequate oral hygiene to patients affected by FOD, including frequent periodontal appointments, prophylaxis, and periodic radiographic follow-up because of the risk of development of other conditions such as simple bone cyst and osteosarcoma. 


\section{References}

1. Kawai T, Hiranuma H, Kishino M, Jikko A, Sakuda M. Cemento-osseous dysplasia of the jaws in 54 Japanese patients. Oral Surg Oral Med Oral Pathol Oral Radiol Endod. 1999;87: 107-114.

2. Neville BW, Albenesius RJ. The prevalence of benign fibroosseous lesions of periodontal ligament origin in Black-skinned women: a radiographic survey. Oral Surg Oral Med Oral Pathol. 1986;62:340-344.

3. Kim JH, Song BC, Kim SH, Park YS. Clinical, radiographic and histological findings of florid cement-osseous dysplasia: a case report. Imaging Sci Dent. 2011;41:139-142.

4. MacDonald-Jankowski DS. Florid cemento-osseous dysplasia: a systematic review. Dentomaxillofac Radiol. 2003;32:141-149.

5. Slootweg PJ. Osseous dysplasia. In: Barnes L, Eveson JW, Reichart P, Sidransky D, eds. WHO Classification of Tumours, Pathology and Genetics of Tumours of the Head and Neck. Lyon, France: International Agency for Research on Cancer (IARC); 2005:323.

6. Alsufyani NA, Lam EWN. Osseous (cemento-osseous) dysplasia of the jaws: clinical and radiographic analysis. J Can Dent Assoc. 2011;77:b70.

7. Speight PM, Carlos R. Maxillofacial fibro-osseous lesions. Curr Diagn Pathol. 2006;12:1-10.

8. Netto JNS, Cerri JM, Miranda AMMA, Pires FR. Benign fibroosseous lesions: clinicopathologic features from 143 cases diagnosed in an oral diagnosis setting. Oral Surg Oral Med Oral Pathol Oral Radiol. 2013;115:E56-E65.

9. Melrose RJ, Abrams AM, Mills BG. Florid osseous dysplasia. A clinical-pathologic study of thirty-four cases. Oral Surg Oral Med Oral Pathol. 1976;41:62-82.

10. Worawongvasu R, Songkampol K. Fibro-osseous lesions of the jaws: an analysis of 122 cases in Thailand. J Oral Pathol Med. 2010;39:703-708

11. Köse TE, Köse OD, Karabas HC, Erdem, Özcan İ. Findings of florid cemento-osseous dysplasia: a report of three cases. J Oral Maxillofac Res. 2014;4:e4.

12. Glascoe A, Brown R, Goode M, Mongelos G. Florid cementoosseous dysplasia and a dental abscess. Dent Today. 2011;30: 102:104-105.

13. MacDonald DS. Maxillofacial fibro-osseous lesions. Clin Radiol. 2015;70:25-36.

14. Sarmento DJS, Monteiro BVB, Medeiros AMC, Silveira EJD. Severe florid cemento-osseous dysplasia: a case report treated conser-vatively and literature review. Oral Maxillofac Surg. 2013;17:43-46.

15. Huh JK, Shin SJ. Misdiagnosis of florid cemento-osseous dysplasia leading to unnecessary root canal treatment: a case report. Restor Dent Endod. 2013;38:160-166.
16. Das BK, Das SN, Gupta A, Nayak S. Florid cemento-osseous dysplasia. J Oral Maxillofac Pathol. 2013;17:150.

17. Lopes MA, Kim HS, Mariano FV, Corrêa MB, Rabelo NT, Vargas PA. Clinico-pathologic conference: case 1. High-grade osteosarcoma (OS) and florid cemento-osseous dysplasia (FCOD). Head Neck Pathol. 2010;4:329-333.

18. Mahomed F, Altini M, Meer S, Coleman H. Cemento-osseous dysplasia with associated simple bone cysts. J Oral Maxillofac Surg. 2005;63:1549-1554.

19. Chadwick JW, Alsufyani NA, Lam EWN. Clinical and radiographic features of solitary and cemento-osseous dysplasia-associated simple bone cysts. Dentomaxillofac Radiol. 2011;40: 230-235.

20. Vieira AJ, Garret JM. Understanding interobserver agreement: the kappa statistic. Fam Med. 2005;37:360-363.

21. Eversole R, Su L, El-Mofty S. Benign fibro-osseous lesions of the craniofacial complex: a review. Head Neck Pathol. 2008;2: 177-202.

22. Muwazi LM, Kamulegeya A. The 5-year prevalence of maxillofacial fibro-osseous lesions in Uganda. Oral Dis. 2015;21: e79e85.

23. Kutluay Köklü H, Çankal DA, Bozkaya S, Ergün G, Barıs, E . Florid cemento-osseous dysplasia: report of a case documented with clinical, radiographic, biochemical and histological findings. $J$ Clin Exp Dent. 2013;5:e58-e61.

24. MacDonald-Jankowski DS. Florid osseous dysplasia in Hong Kong Chinese. Dentomaxillofac Radiol. 1996;25:39-41.

25. MacDonald-Jankowski DS. Gigantiform cementoma occurring in two populations, London and Hong Kong. Clin Radiol. 1992;45: 316-318.

26. Schneider LC, Dolinsky HB, Grodjesk JE, et al. Malignant spindle cell tumor arising in the mandible of a patient with florid osseous dysplasia. Oral Surg Oral Med Oral Pathol Oral Radiol Endod. 1999;88:69-73.

27. Melrose R, Handlers J. Osteosarcoma ex florid osseous dysplasia: report of a case. Oral Surg Oral Med Oral Pathol. 2003;96:296.

\section{Reprint requests:}

Pablo Agustin Vargas, DDS, PhD, FRCPath Faculdade

de Odontologia de Piracicaba - UNICAMP

Departamento de Diagnóstico Oral - Semiologia Av.

Limeira

901 CEP 13.414-903

Piracicaba

São Paulo

Brazil

Pavargas@fop.unicamp.br 